\title{
A Preliminary Study of Three Finishing Materials for Traditional Chinese Furniture
}

\author{
Xin You Liu, Timar Maria Cristina, Varodi Anca Maria \\ Transilvania University, Brasov, Romania \\ Email: liu.xinyou@unitbv.ro \\ Received 3 April 2014; revised 8 May 2014; accepted 15 May 2014 \\ Copyright $@ 2014$ by authors and Scientific Research Publishing Inc. \\ This work is licensed under the Creative Commons Attribution International License (CC BY). \\ http://creativecommons.org/licenses/by/4.0/

(c) (1) Open Access

\begin{abstract}
The traditional Chinese furniture evolved as an important part of the Chinese culture and civilization. Alongside design, structure, ornaments, the original finishes applied define the particularities and value of traditional Chinese furniture. This is because wood finishing not only enhances wood natural beauty by colour, gloss and highlighting of wooden texture, but also can be a means of exquisite decoration (e.g. engraved, gilded or painted lacquer works). Chinese lacquer, Tung oil and Chinese wax represent some of the most important finishing materials employed and were, therefore, selected for this study. The paper presents a brief macroscopic, microscopic and FTIR characterization of these materials. These results will be used as starting data in a further study on the ageing properties of these traditional finishing materials, useful for scientific conservation purposes.
\end{abstract}

\section{Keywords}

Traditional Chinese Finishing Materials, Chinese Lacquer, Chinese Wax, Tung Oil, Microscopy, FTIR

\section{Introduction}

The traditional Chinese furniture evolved as an important part of the Chinese culture and civilization [1]. The history of the traditional Chinese furniture is divided into four historical periods as follows: the Youth period (possible BC 5000 - BC 770), the Growth period (BC 770 - AD 220), the Development period (AD 220 - AD 979) and the Maturity period (AD 960 - AD 1911) [2]. Several dynasties are included in each of these periods, as detailed in a previous publication [3]. Among these it is worth mentioning for instance the Ming Dynasty (1368 - 1644) in the Maturity period, when it is considered that Chinese furniture reached a peak of development in its multi-millenary existence [4]. 
Alongside design, structure, ornaments, wood species, the finishes of wooden surfaces define the particularities and value of traditional Chinese furniture. This is because wood finishing not only enhances wood natural beauty by colour, gloss and highlighting of wooden texture, but also can be a means of exquisite decoration (e.g. engraved, gilded or painted lacquer works). Moreover, some finishes may impart outstanding time resistance to the wooden objects (e.g. lacquer ware from 5000 BC [11]).

Chinese lacquer, Tung oil and Chinese wax represent some of the most important finishing materials employed and were, therefore, selected for this study. Figure 1 presents a scheme showing the time sequence of the four periods in the history of Chinese furniture and the employment of the selected wood finishing materials within these periods.

Chinese lacquer is a natural, very durable finishing material. This is actually the sap of some lacquer trees, such as Rhus vernicifera, which is tapped (raw Urushi lacquer) and further processed to produce different lacquer sorts (e.g. Kurome Urushi) (Figure 2(a)). The high resistance of the lacquered surfaces is related to its complex chemical composition and the chemical mechanism of curing [12].

Tung oil is a siccative (drying) oil extracted from the seeds of a group of wood species (around 9), generically called Tung trees (Figure 2(b)), such as Vernicia montana (Lour.) and Vernicia fordii (Hemsl.). The ratio of

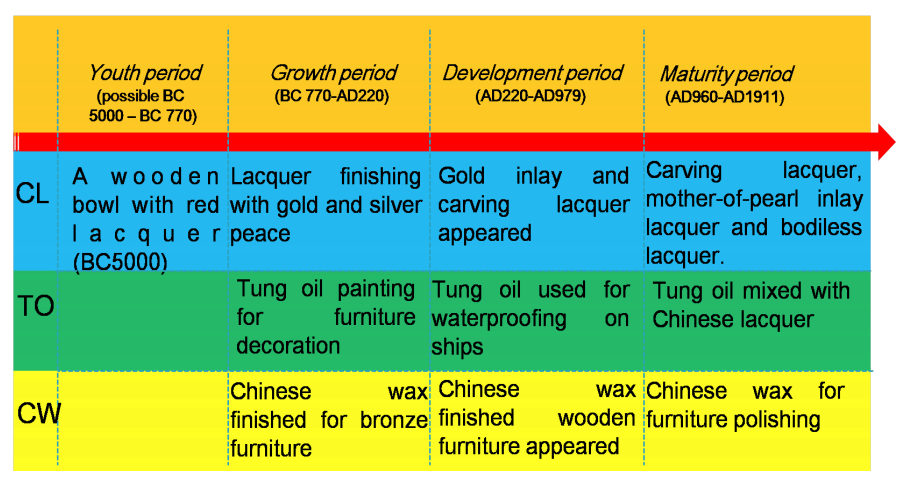

Figure 1. Periods in the history of traditional Chinese furniture and time sequences for the utilization of three Chinese finishing materials: Chinese lacquer (CL), Chinese wax (CW), Tung oil (TO) - compilation of data from References [5]-[12].
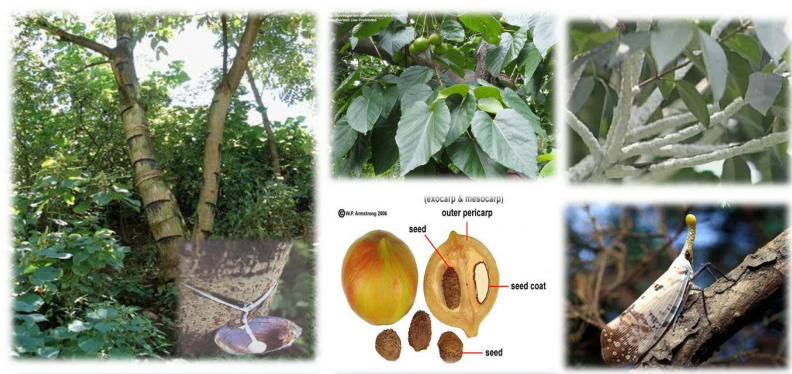

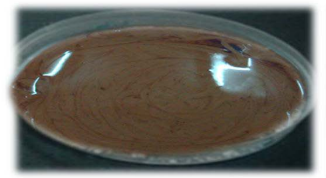

(a)

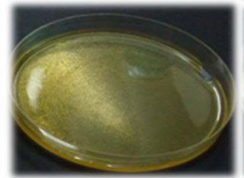

(b)

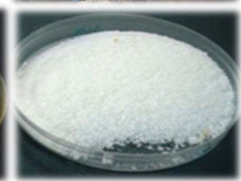

(c)

Figure 2. Sources and aspect of the three studied Chinese finishing materials: (a) lacquer tree (Rhus vernicifera) and raw Chinese lacquer; (b) Tung tree (Vernicia montana), seeds and Tung oil; (c) wax tree (Fraxinus chinensis), wax insect (Ericerus pela) and Chinese wax (images processed after [15]-[17] and experimental products). 
Tung oil in the dried source seeds may reach a maximum percent of $68 \%$ [13]. It has been extensively used for transparent wood finishing, painting and varnishes. The fast "drying" process and the resistance of the finished surfaces are related to its chemistry [14].

Chinese wax is a sort of insect wax, secreted by Coccus ceriferus or Ericerus pela, which deposite it on the twigs of some species of ash trees (Ligustrum lucidum Ait. or Fraxinus chinensis) (Figure 2(c)). It is a white to yellowish-white, gelatinous, crystalline water-insoluble substance, with a complex chemical composition [9] with different applications including wood finishing.

\section{Research Objectives}

The present paper is a part of a $\mathrm{PhD}$ research project referring to a comparative study of ageing phenomena of wooden support and transparent traditional finishes for European and Chinese furniture.

The current paper covers some preliminary steps within this project, namely:

- A brief presentation of three important Chinese traditional finishing materials as origin and basic properties based on literature data;

- Their experimental macroscopic, microscopic and FTIR investigation.

The resulted data will be used as reference for the further ageing tests to be carried on.

\section{Materials and Methods}

The three finishing materials selected for this study were purchased from China: Chinese lacquer from East Lake CO., Ltd., Tung oil from Jin Xing Chemical and Chinese wax from Heng Hong Feng La Jiao Ye CO., Ltd.

The macroscopic aspect of the products was observed directly and pictures were taken on samples placed in Petri dishes employing a digital camera (Sony W80).

In order to observe the macroscopic aspect and the microscopic features of resulting dried coating films, the products were applied on microscopic glass lamellas and allowed to dry in normal conditions. Chinese lacquer (viscous liquid) and Tung oil (fluid liquid) were applied (after homogenisation) with a spatula as thin films of about $30-50 \mu \mathrm{m}$. In the case of Chinese wax (solid product) a small pellet was put on a glass lamella which was placed on a heating plate at $50^{\circ} \mathrm{C}$ and allowed to melt and flow freely to form an uniformous thin film and further cooled down at room temperature for re-solidification. The microscopic lamellas were examined under an optical stereomicroscope OPTIKA SZM fitted with an image capture system (OPTIKAM PRO3 video camera), in transmitted light (TL). For the Tung oil a dark field (DF) device was employed in order to increase contrast. From each sample there were taken several images at magnifications of $40 \times$ and $80 \times$.

FTIR investigation was performed on the same film samples applied on glass lamellas which were previously observed under microscope. FTIR spectra were recorded employing an ALPHA FT-IR Spectrometer (BRUKER), equipped with ATR (attenuated total reflection) module. The spectra were recorded in the $4000-400 \mathrm{~cm}^{-1}$ at a resolution of $2 \mathrm{~cm}^{-1}$, each spectra representing an average of 32 scans.

\section{Results and Discussion}

The macroscopic aspect of the products can be observed in the pictures included at the bottom of Figure 2. Chinese lacquer is a viscous whitish (milky to cream colour) liquid with sour smell, which colour turns to light brown when exposed to open air. The Tung oil is a homogenous liquid with light yellow colour and characteristic smell. Chinese wax is white solid, with a specific structure, including opaque amorphous material and crystalline, translucent, high reflective, granules.

The dry coating films obtained on glass lamellas could be characterised as follows: dark brown, opalescent, glossy (Chinese lacquer), yellowish, transparent, and glossy (Tung oil), whitish, opalescent, satinated (Chinese wax).

Their microscopic investigation revealed characteristic differentiating features, as can be observed in Figure 3. For the Chinese lacquer film (Figure 3(a) and Figure 3(b)) is characteristic a quite regular microscopic structure with a certain roughness, in which can be observed dark brown insular areas (mostly of around 20 - 40 $\mu \mathrm{m})$ on a more uniform whitish film with micro-crystalline structure. This is in accordance to studies of Takayuki Honda et al. (2008) [18] and explainable by the complex composition of this material.

A characteristic microstructure, with wavy aspect, could be distinguished also for the Tung oil film when 


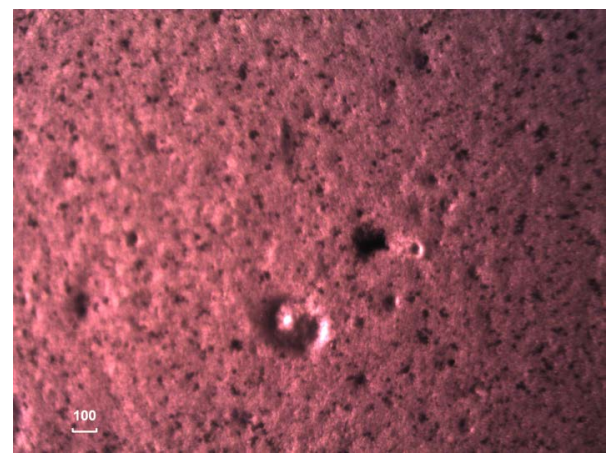

(a)

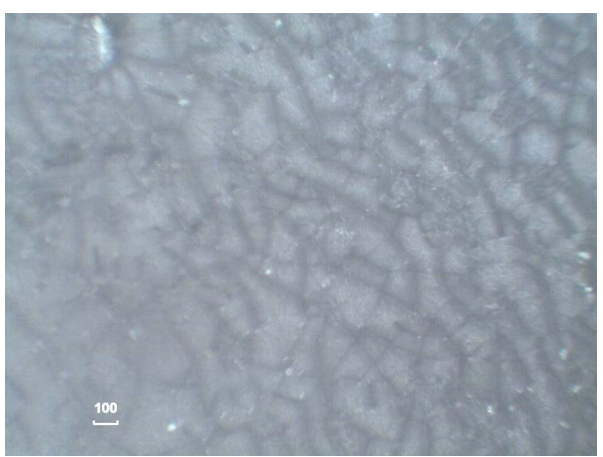

(c)

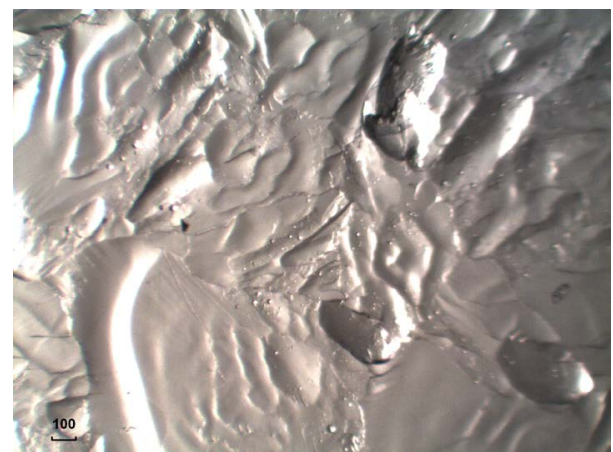

(e)

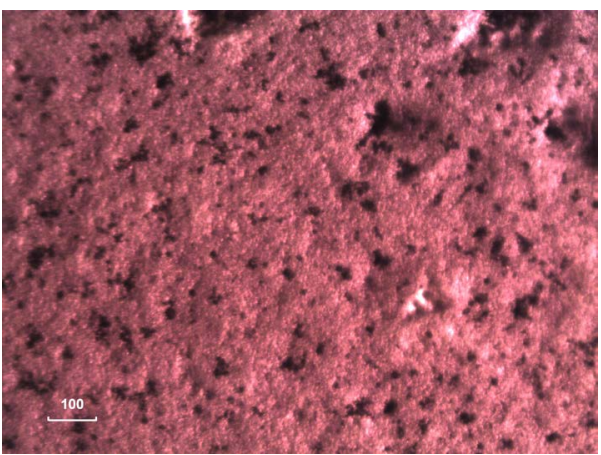

(b)

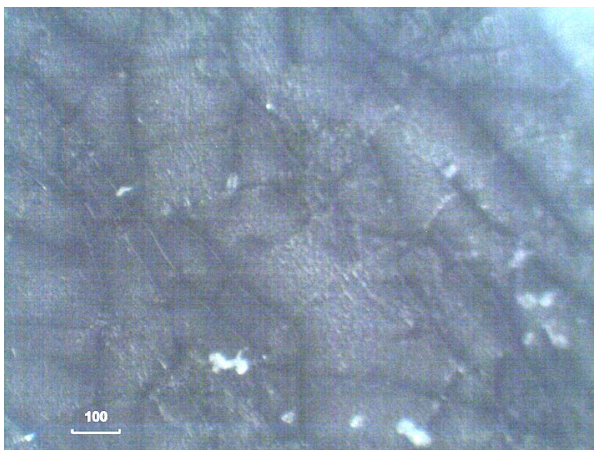

(d)

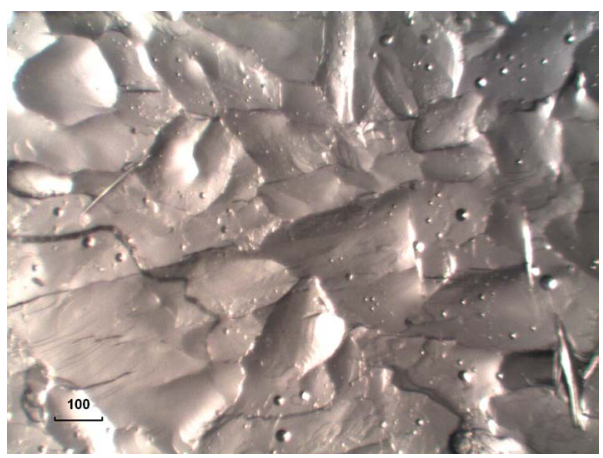

(f)

Figure 3. Micrographs of the films on glass lamellas(unit is $\mu \mathrm{m}$ ): (a) Chinese lacquer $40 \times$ magnification; (b) Chinese lacquer $80 \times$ magnification; (c) Tung oil 40× magnification; (d) Tung oil 80× magnification; (e) Chinese wax 40× magnification; (f) Chinese wax 80× magnification.

studied in dark field (Figure 3(c) and Figure 3(d)). Chinese wax forms a high reflective film with a specific less regular pattern (wavy areas alternating with geometrical/rhomboidal areas of $100-200 \mu \mathrm{m}$ ), as can be observed in Figure 3(e) and Figure 3(f).

In Figure 4 are presented the FTIR-ATR spectra experimentally recorded for the studied products, while in Tables 1-3 are summarised the main absorbance bands found in these spectra and their assignment in accordance to literature. To facilitate understanding of bands assignment to different functional groups/structural elements, the chemical structure of the main components of these finishing products, as presented in literature, is also included.

The comparative analysis of the spectra and associated tables allows a differentiation of these products based on their characteristic chemical features, which include both common and specific structural elements. For instance, the phenol type structure of the Chinese lacquer is well revealed by a strong - OH band at around 3400 $3600 \mathrm{~cm}^{-1}$, while the ester type structure of Tung oil and Chinese wax explain the strong $\mathrm{C}=\mathrm{O}$ band at around $1700-1740 \mathrm{~cm}^{-1}$. All the three products contain in their structure long aliphatic chains, presenting therefore 


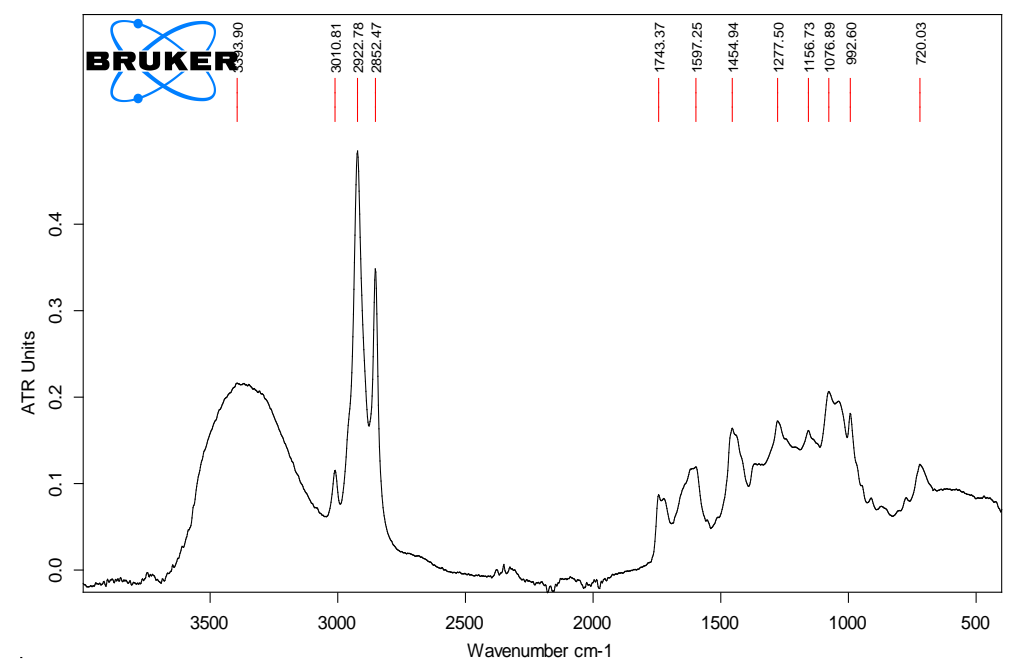

(a)

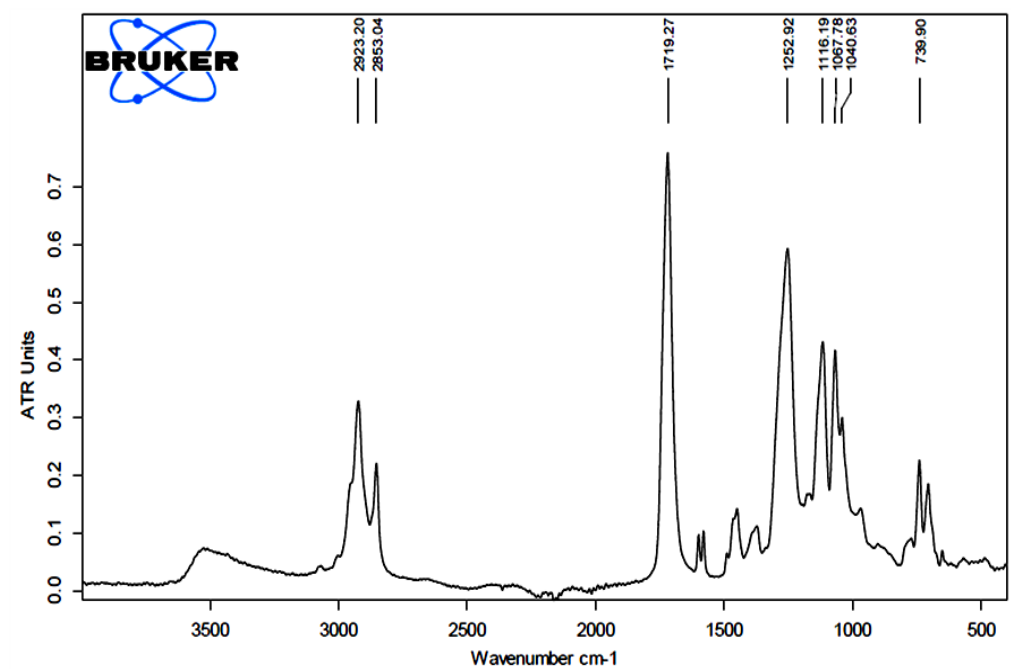

(b)

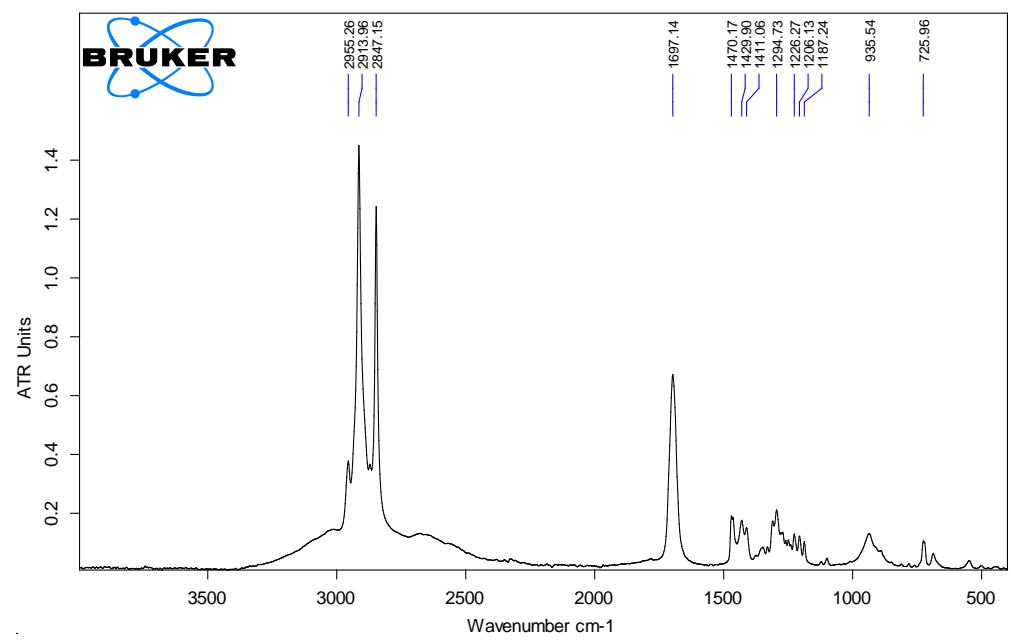

(c)

Figure 4. The FTIR-ATR spectra of three finishing materials: (a) Chinese lacquer; (b) Tung oil; (c) Chinese wax. 
Table 1. Bands assignment for the FTIR spectra of Chinese lacquer (experimental vs. literature).

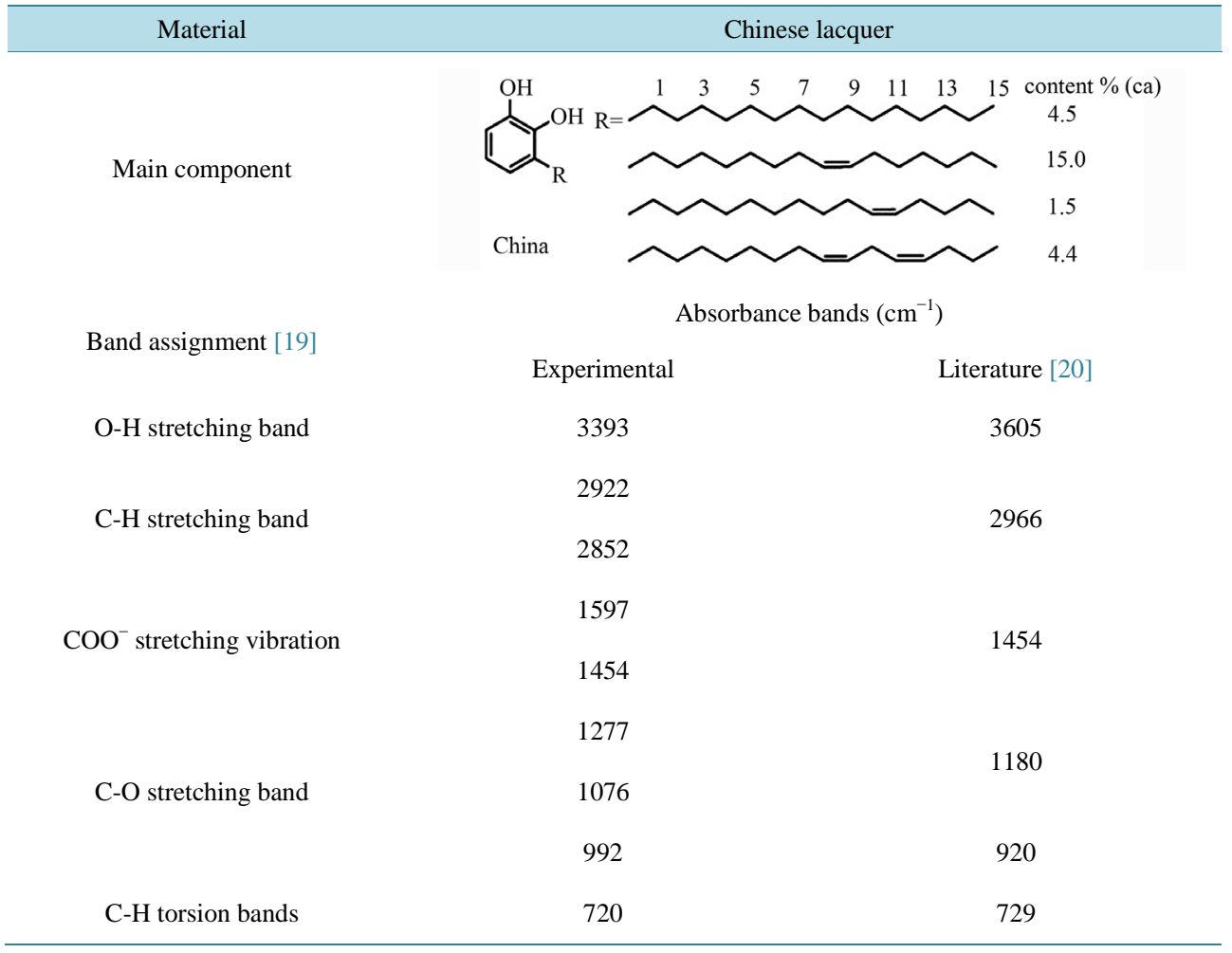

Table 2. Bands assignment for the FTIR spectra of Tung oil (experimental vs. literature).

\begin{tabular}{|c|c|c|}
\hline Material & \multicolumn{2}{|c|}{ Tung oil } \\
\hline Main component & \multicolumn{2}{|c|}{$\begin{array}{l}\mathrm{CH}_{3}\left(\mathrm{CH}_{2}\right)_{3}-\mathrm{CH}=\mathrm{CH}-\mathrm{CH}=\mathrm{CH}-\mathrm{CH}=\mathrm{CH}-\left(\mathrm{CH}_{2}\right)_{7}-\mathrm{COOCH} \mathrm{H}_{2} \\
\mathrm{CH}_{3}\left(\mathrm{CH}_{2}\right)_{3}-\mathrm{CH}=\mathrm{CH}-\mathrm{CH}=\mathrm{CH}-\mathrm{CH}=\mathrm{CH}-\left(\mathrm{CH}_{2}\right)_{7}-\mathrm{COOCH}\end{array}$} \\
\hline \multirow{2}{*}{ Band assignment [19] } & \multicolumn{2}{|c|}{ Absorbance bands $\left(\mathrm{cm}^{-1}\right)$ [14] } \\
\hline & Experimental & Literature \\
\hline C-H stretching band & $\begin{array}{l}2923 \\
2853\end{array}$ & $\begin{array}{l}3020 \\
2930\end{array}$ \\
\hline $\mathrm{C}=\mathrm{O}$ stretching band & 1719 & 1750 \\
\hline \multirow{2}{*}{$\mathrm{COO}^{-}$stretching vibration } & 1562 & 1550 \\
\hline & - & 1452 \\
\hline C-O stretching band & $\begin{array}{l}1116 \\
1067\end{array}$ & 990 \\
\hline C-H torsion bands & 739 & - \\
\hline
\end{tabular}

strong characteristic CH absorption bands (as a two peaks band) at around $2900 \mathrm{~cm}^{-1} / 2850 \mathrm{~cm}^{-1}$. The recorded spectra and data on characteristic bands are in good accordance to literature. 
Table 3. Bands assignment for the FTIR spectra of Chinese wax (experimental vs. literature).

\begin{tabular}{ccc}
\hline Material & \multicolumn{2}{c}{ Chinese wax } \\
\hline Main component & \multicolumn{2}{c}{$\mathrm{CH}_{3}\left(\mathrm{CH}_{2}\right)_{25} \mathrm{COO}\left(\mathrm{CH}_{2}\right)_{25} \mathrm{CH}_{3}$} \\
Assignment [19] & \multicolumn{2}{c}{ Absorbance bands $\left(\mathrm{cm}^{-1}\right)$} \\
& Experimental & Literature [19] \\
C-H stretching band & 2913 & 2918 \\
& 2847 & 2849 \\
C=O stretching band & 1679 & 1734 \\
COO- stretching vibration & 1470 & 1466 \\
& 1226 & 1182 \\
C-O stretching band & 935 & 724 \\
\hline
\end{tabular}

\section{Conclusion}

Three traditional finishing materials important for Chinese historic furniture: Chinese lacquer, Tung oil and Chinese wax, were comparatively investigated. Optical microscopy revealed characteristic microscopic features of the resulting coating films, while the FTIR spectra highlighted both the common and differentiating chemical structure features. These FTIR spectra will be used as references in a further study on the ageing properties of these finishing materials and finished wooden surfaces. This is important for scientific conservation of valuable historic Chinese furniture or other old wooden artefacts.

\section{References}

[1] Weihua, Z. (2011) A Research into the Traditional Chinese Paintings (the Ruler Paintings) and the Ancient Furniture Culture. 12th International Conference on Computer-Aided Industrial Design \& Conceptual Design, Chongqing, 27-29 November 2011, 1046-1050.

[2] (2014) The history of Chinese furniture http://wenku.baidu.com/link?url=w70VBTF2LJG4w6XAOUrkiJntYCPoS7WOXCV2SP5eykN6LGiaO6aZwg41dh5S 4VK-xecU9NxqMfFGvexCjIldxUmtOtxXYUv4EmprilVuOnG

[3] Liu, X.Y. and Timar, M.C. (2013) A Study on the History and Materials of Traditional Chinese Furniture. The 9th International Conference "Wood Science and Engineering in the Third Millenium", Brasov, 7 November 2013, 256-264.

[4] Berliner, N. (1996) Beyond the Screen: Chinese Furniture of the 16th and 17th Centuries. Museum of Fine Arts, Boston, 81-117.

[5] Chena, Y.-H., Chena, J.-H., Changb, C.-Y. and Changb, C.-C. (2010) Biodiesel Production from Tung (Vernicia montana) Oil and Its Blending Properties in Different Fatty Acid Compositions. Bioresource Technology, 101, 9521-9526. http://dx.doi.org/10.1016/j.biortech.2010.06.117

[6] Guan, C.Y. (1999) A Preliminary Study of Chinese Tung Tree Planting History. Ancient and Modern Agriculture, No. 4, 21-28.

[7] Hang, J. And Guo, Q.H. (2006) Chinese Art \& Crafts. China Intercontinental Press, Beijing, 58-64.

[8] Greenfield, J. (1959) Lectures of the 1959 Short Course on Drying Oils. The Journal of The American Oil Chemists' Society, 36, 565-574.

[9] Da, T. (2007) The Inherited and Application of Waxing Technique for Ming-Style Furniture. Chinese Furniture, No. $10,60-61$.

[10] Tan, G.H. (2005) The Raw Chinese Lacquer Utilization and Inspection. Journal of Chinese Lacquer, 24, 44-48.

[11] Tian, T. (2007) Chinese Lacquer Art. China \& the World Cultural Exchange, Beijing, 20-22.

[12] Zhou, B.Z. (1985) The Protection of Ancient Chinese Lacquerware. Urushi Study Group, Tokyo, 71-78.

[13] Pu, X., Zhang, X.H., Tong, S.L. and Xue, P. (2003) Study and Application Prospect of Modified Tung Oil. Forest Chemical Communications, 37, 41-46. 
[14] Li, H.Y. (2009) Scientific Research on Traditional Tung Oil Boiling Technology. Master Thesis, Shan Xi Normal University, Linfen, 22-25.

[15] (2014) Vietnamese Lacquerware. http://www.3pl.asia/index.php?route=information/information\&information_id=6

[16] (2014) Tung Oil Tree. http://waynesword.palomar.edu/tungoil1.htm

[17] (2014) How to feed wax insect. http://www.cndfilm.com/special/xhj2012/20130311/105038.shtml

[18] Takayuki, H., Rong, L., Risa, S., Takahisa, I. and Tetsuo, M. (2008) Characterization and Comparison of Asian Lacquer Saps. Progress in Organic Coatings, 61, 68-75. http://dx.doi.org/10.1016/j.porgcoat.2007.09.003

[19] Michele, R., et al. (1999) Infrared Spectroscopy in Conservation Science. The Getty Conservation Institute, Los Angeles, 99-199.

[20] Jin, Z.Y. and Chen, T.Y. (1985) Analysis of FTIR Spectra of Chinese Lacquer. Journal of Chinese Lacquer, 1, 1-10. 